\title{
Assessing the Prevalence of Mechanical Dyssynchrony with Activity in Patients with Low Ejection Fraction and Narrow QRS at Rest
}

Sachin Kumar Jain, Timothy R. Larsen, Peter Burke, Dustin Feldman and Christian Machado*

Chief of Electrophysiology, Providence Heart Institute, Providence Hospital and Medical Center Cardiology, USA

\begin{abstract}
Introduction: Cardiac Resynchronization Therapy (CRT) improves hemodynamics, symptoms, and overall mortality in patients with advanced heart failure (HF) and ventricular electrical dyssynchrony (QRS duration $>120$ $\mathrm{msec}$ ). Previous studies have shown that mechanical dyssynchrony (MD) may be present in up to $45 \%$ of patients with advanced HF and QRS duration $<120 \mathrm{~ms}$ at rest. We determined whether activity induces MD in patients with QRS duration $<120$ msec.

Methods: A total of 47 consecutive patients with left ventricular ejection fraction (LVEF) $\leq 30 \%$, New York Heart Association (NYHA) class II-IV HF, and a QRS complex $<120$ ms were evaluated for MD at rest and exercise utilizing a modified Bruce protocol and three dimensional echocardiography. Time to peak systolic velocity was measured via Tissue Doppler and MD was defined as a time delay of $>65 \mathrm{msec}$ from peak systolic activation of the septal wall to the lateral wall of the left ventricle. Minnesota living with heart failure questionnaire (MLWHFq), EF and NYHA class were assessed to determine risk factors for exercise induced MD.

Results: Of the 47 patients, MD occurred in 11 patients (23\%) at rest and 5 patients (13\%) at exercise. The mean time to peak systolic velocity in the rest and exercise dyssynchrony groups was $105 \pm 32 \mathrm{msec}$ and 124 \pm 29 msec respectively, compared with $45+/-15$ msec in patients not experiencing dyssynchrony. No patients experienced electrical dyssynchrony with activity. EF, NYHA class or MLWHF questionnaire were not predictive.

Conclusion: MD with activity is not uncommon in patients with HF and a narrow QRS. MD should consider including patients with exercise induced MD as this population otherwise may go ignored. Additionally, patients with pre-existing electrical dyssynchrony who develop MD with exercise may benefit from optimization of their device settings to meet the potential hemodynamic challenge rendered by increased physical activity and heart rate.
\end{abstract}

Keywords: Cardiac resynchronization therapy; Mechanical dyssynchrony; Low ejection fraction; Narrow QRS at rest

\section{Introduction}

Heart failure with systolic impairment is currently treated with a combination of medications and implantable devices. One such implantable device is the biventricular pacemaker. Biventricular pacemakers pace both the right and left ventricles simultaneously (as opposed to traditional pacemakers which pace from the right ventricle only). This approach is known as cardiac resynchronization therapy (CRT) [1,2]. According to current guidelines CRT should be considered in patients with New York Heart Association (NYHA) class II-IV symptoms, impaired systolic function (left ventricular ejection fraction <30-35\%), and interventricular conduction delay (QRS duration of $>120 \mathrm{msec}$ ) [3]. The benefit of CRT has been most clearly demonstrated for patients with electrical dyssynchrony (i.e. QRS duration $>120 \mathrm{msec}$ ), there are, however, a large proportion of patients with normal QRS duration yet evidence of mechanical dyssynchrony (MD) either at rest or upon exertion [4,5]. The utility of CRT in this population is less well defined.

\section{Methods}

Patients were identified as they presented to the Heart Institute at Providence Hospital for insertion of an implantable cardioverter defibrillator (ICD) for primary prevention of sudden cardiac death over a 3 month period. Patients with left ventricular systolic dysfunction and ejection fraction (LVEF) $<30 \%$, symptoms consistent with New York Heart Association class II-IV, and QRS duration $<120 \mathrm{msec}$ on resting electrocardiogram were included. Written informed consent was obtained from all patients. Patients unwilling or unable to give informed consent or with contraindications to exercise stress testing were excluded. This study was approved by the Investigation Review Board of Providence Hospital and Medical Center.

Basic demographics, past medical history, and current medical therapies were collected prospectively at the time of enrollment. Medical therapy for heart failure was determined by the patient's primary treating physician. At the time of enrollment New York Heart Association classification was determined and patients completed the Minnesota living with heart failure questionnaire (MLWHFq).

Mechanical dyssynchrony was evaluated with real time 3-dimential echocardiography. There are several ways to determine the mechanical dyssynchrony. But we chose to tissue Doppler difference in peak systolic velocity between two walls, because we felt it is easy to measure, reliable and reproducible. The time to peak systolic velocity was measured with Tissue Doppler. Mechanical dyssynchrony was defined as a time to peak systolic velocity delay greater than $65 \mathrm{msec}$ from the earliest to

*Corresponding author: Christian E Machado, Chief of Electrophysiology, Providence Heart Institute, Providence Hospital and Medical Center Cardiology 16001 W Nine Mile Rd, Southfield, MI 48075, USA, Tel: 12489611758; E- mail: cmachado@comcast.net

Received November 28, 2013; Accepted December 30, 2013; Published January 06, 2014

Citation: Jain SK, Jain A, Larsen TR, Burke P, Feldman D (2014) Assessing the Prevalence of Mechanical Dyssynchrony with Activity in Patients with Low Ejection Fraction and Narrow QRS at Rest. J Cardiovasc Dis Diagn 2: 138 doi:10.4172/2329-9517.1000138

Copyright: (C) 2014 Jain SK, et al. This is an open-access article distributed under the terms of the Creative Commons Attribution License, which permits unrestricted use, distribution, and reproduction in any medium, provided the original author and source are credited. 
Citation: Jain SK, Jain A, Larsen TR, Burke P, Feldman D (2014) Assessing the Prevalence of Mechanical Dyssynchrony with Activity in Patients with Low Ejection Fraction and Narrow QRS at Rest. J Cardiovasc Dis Diagn 2: 138. doi:10.4172/2329-9517.1000138

the latest ventricular wall segment (typically the septal wall and lateral wall of the left ventricle) activation using a standard 12-segment model.

All patients underwent assessment of mechanical dyssynchrony at rest and with exercise. Exercise evaluation was performed using a standard treadmill exercise stress test with a modified Bruce protocol. The patients walked on the treadmill on symptom limited modified Bruce protocol. The indication for the stoppage of the exercise were achievement of the peak heart rate, fatigue, hypotension, significant arrhythmias or anginal chest pain. As per protocol, the 3D echocardiogram was obtained as early as possible after completion of the exercise portion of the stress test and the patient is able to lie back on the table (Within 10 to 15 seconds).

\section{Results}

A total of 47 patients completed the study protocol. Of these 47 patients $20(43 \%)$ had diabetes mellitus, $28(60 \%)$ had documented coronary artery disease, 39 (83\%) had hypertension, and $13(28 \%)$ had chronic kidney disease stage III or worse (glomerular filtration rate $<60$ $\left.\mathrm{mL} / \mathrm{min} / 1.73 \mathrm{~m}^{2}\right)$. In total $45(96 \%)$ patients were taking an angiotensin system inhibitor, 45 (96\%) were taking a beta-blocker, 43 (91\%) were taking an aldosterone antagonist, and 38 (81\%) were taking a statin.

Of the 47 patients who completed the study protocol $11(23 \%)$ demonstrated MD at rest. Of the remaining 36 patients 5 (13\%) demonstrated $\mathrm{MD}$ with exertion. No patients experience electrical dyssynchrony (QRS duration $>120 \mathrm{msec}$ ). There were no significant differences in baseline demographics, MLWHFq score, or NYHA class among patients who did and who did not demonstrate MD at rest or with exercise. There were no differences in dyssynchrony between ischemic vs. non ischemic cardiomyopathy patients (Data not shown). The most common reason for stopping the exercise stress test in our cohort was reaching peak heart rate and patient fatigue. There were no differences noted among the different NYHA class patients. (Data not shown)
Of the patients who experienced MD at rest 7 (63\%) had NYHA class II symptoms, $3(60 \%)$ patients with MD only on exertion had NYHA class II symptoms, 20 (64\%) patients without MD had NYHA class II symptoms, the remaining patients had NYHA class III or IV symptoms. The MLWHFq score for patients with MD at rest was 39 \pm 5 , MD with activity $33 \pm 7$, and no MD $41 \pm 4$ (Figure 1) (Tables 1 and 2).

\section{Discussion}

A total of 47 consecutive patients were evaluated for dyssynchrony at rest and with exercise. There were $11(23 \%)$ patients that experienced dyssynchrony at rest. Among the cohort, $14 \%(n=5)$ of patients experienced mechanical dyssynchrony with activity. No patients experienced electrical dyssynchrony with activity. There were no significant differences in demographics, MLWHF questionnaire, and NYHA class among patients that experienced mechanical dyssynchrony with exercise and those that did not.

Cardiac resynchronization therapy (CRT) improves hemodynamics and symptoms in patients with advanced heart failure (HF) and myocardial dyssynchrony [6]. The proposed mechanism includes both improved pump efficiency and reversal of deleterious myocardial remodeling. Results from mechanistic studies, observational evaluations, randomized controlled trials and their meta-analyses have consistently demonstrated significant improvement in quality of life, functional status, exercise capacity, hospitalization, and mortality in patients with New York Heart Association (NYHA) class III and IV HF and electrical dyssynchrony who are randomized CRT [7]. More recently the MADIT-CRT trial demonstrated improvement in all cause mortality and progression of heart failure in patients with NYHA class I and II, prompting expansion of CRT indications to include all patients with heart failure with impaired systolic function and left bundle branch block morphology on EKG [8].

There is a growing body of evidence demonstrating mechanical dyssynchrony in patients with normal QRS duration $(<120 \mathrm{msec})$. The

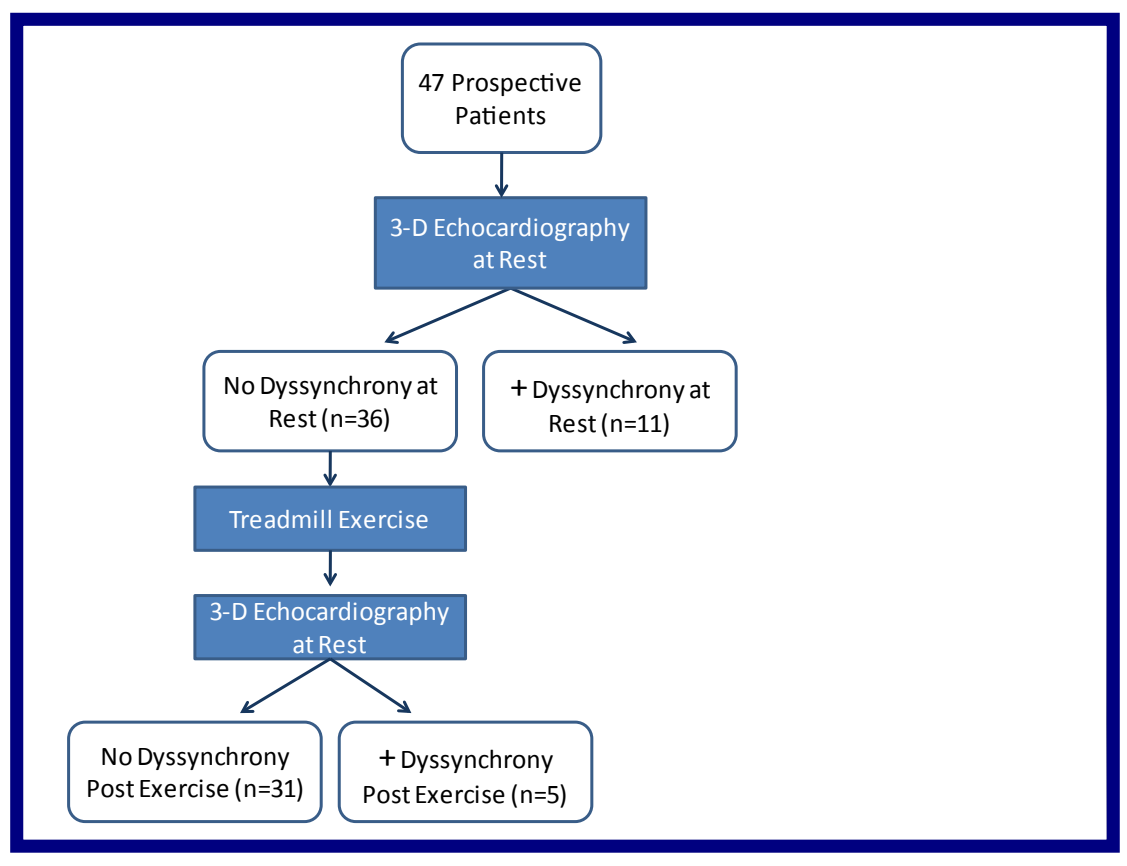

Figure 1: Patient Flow Through Study. 
Citation: Jain SK, Jain A, Larsen TR, Burke P, Feldman D (2014) Assessing the Prevalence of Mechanical Dyssynchrony with Activity in Patients with Low Ejection Fraction and Narrow QRS at Rest. J Cardiovasc Dis Diagn 2: 138. doi:10.4172/2329-9517.1000138

Page 3 of 4

\begin{tabular}{|c|c|c|c|c|c|}
\hline & No Dyssynchrony at Rest (36) & Dyssynchrony at Rest (11) & No Dyssynchrony with Activity (31) & Dyssynchrony with Activity (5) & $\mathrm{p}$ Value \\
\hline $\mathrm{DM}$ & $42 \%(15)$ & $45 \%(5)$ & $48 \%(15)$ & $40 \%(2)$ & NS \\
\hline CAD & $61 \%(22)$ & $54 \%(6)$ & $52 \%(16)$ & $80 \%(4)$ & NS \\
\hline HTN & $86 \%(31)$ & $72 \%(8)$ & $81 \%(25)$ & $80 \%(4)$ & NS \\
\hline CKD (GFR <60) & $25 \%(9)$ & $36 \%(4)$ & $32 \%(10)$ & $40 \%(2)$ & NS \\
\hline Statin & $86 \%(31)$ & $63 \%(7)$ & $77 \%(24)$ & $40 \%(2)$ & NS \\
\hline NYHA class I-II & $64 \%(23)$ & $63 \%(7)$ & $64 \%(20)$ & $60 \%(3)$ & NS \\
\hline ACE/ARB & $94 \%(34)$ & $100 \%(11)$ & $97 \%(30)$ & $100 \%(5)$ & NS \\
\hline ßeta-Blockers & $97 \%(35)$ & $91 \%(10)$ & $100 \%(30)$ & $80 \%(4)$ & NS \\
\hline MLWHFq & $31 \pm 5$ & $39 \pm 5$ & $41 \pm 4$ & $33 \pm 7$ & NS \\
\hline Mean LVEF (\%) & $27 \pm 3$ & $29 \pm 5$ & $28 \pm 6$ & $29 \pm 3$ & NS \\
\hline $\begin{array}{l}\text { Aldosterone } \\
\text { Antagonists }\end{array}$ & $92 \%(33)$ & $91 \%(10)$ & $90 \%(28)$ & $80 \%(4)$ & NS \\
\hline
\end{tabular}

Table 1: Distribution of Baseline Characteristics (n).

\begin{tabular}{|l|l|l|l|}
\hline & $\begin{array}{l}\text { No Dyssynchrony at Rest } \\
(n=36)\end{array}$ & Dyssynchrony at Rest ( $=11)$ & $\begin{array}{l}\text { No Dyssynchrony with } \\
\text { Activity } \\
(n=31)\end{array}$ \\
$\begin{array}{l}\text { (Ts-12) Mean Time to Peak Systolic } \\
\begin{array}{l}\text { Velocity } \\
(\mathrm{msec})\end{array}\end{array}$ & $45 \pm 15 \mathrm{msec}$ & $105 \pm 32 \mathrm{msec}$ & $31 \pm 18 \mathrm{msec}$ \\
\hline
\end{tabular}

Table 2: Mechanical Dyssynchrony defined by Ts-12.

prevalence in patients with NYHA class III or IV symptoms and normal QRS duration appears to be around 25-47\% [4,9]. There is conflicting evidence regarding the benefit of CRT in this patient population with several studies suggesting clinical improvement with reversal of remodeling and one randomized controlled trial, however, found no clinical benefit [10-12]. The NARROW-CRT trial demonstrated improvement in heart failure symptoms without change in heart failure hospitalization or death in this patient population [13]. But the most recent study Cardiac Resynchronization Therapy in Heart Failure with a Narrow QRS Complex, which was halted early for futility by data and safety monitoring board. It concluded in patients with systolic heart failure and QRS duration of less than $130 \mathrm{msec}$, CRT does not reduce the rate of death or hospitalization for heart failure and may increase mortality [13].

Our current analysis identified an incidence of $\mathrm{MD}$ at rest of $23 \%$ (on the low end of previous estimates). Prior studies assessed dyssynchrony at rest and thus may miss a subgroup of patients who have no dyssynchrony at rest but may develop dyssynchrony with activity. In our study group 5 patients (14\%) developed MD with activity. It is unknown whether or not CRT would be of benefit in these patients. This thus identifies a subpopulation requiring further study regarding the utility of CRT. Additionally, patients with preexisting electrical dyssynchrony who develop MD with exercise may benefit from optimization of their device settings to meet the potential hemodynamic challenge rendered by increased physical activity and heart rate.

A recent case study describes a patient with a narrow QRS complex without LV dyssynchrony who experienced an acute exacerbation of HF following exercise. Analysis revealed that an increase in heart rate induced acceleration-dependent LBBB with severe LV dyssynchrony followed by acute $\mathrm{HF}$ and hemodynamic collapse. CRT prevented these adverse reactions $[14,15]$. Further research will determine if the optimal evaluation for CRT should include testing for LV dyssynchrony not only at rest, but also during exercise as to not miss this subgroup of patients who may benefit from therapy.

There are several possible explanations for the exercise induced MD causing hemodynamic instability. Firstly it could be that an increase in heart rate on the already compromised left ventricle and its cardiac output. Secondly exercise induced rate dependent LBBB with severe left ventricular dyssynchrony followed by acute decompensation, heart failure and hemodynamic collapse (which none of our cohorts had). Thirdly, the theoretical possibility of inhomogeneous mechanical contractility because of worsening mitral regurgitation with exercise, increasing left atrial pressure, thereby more volume into left ventricle, increasing left ventricular end diastolic pressure, thus causing exercise induced mechanical dyssynchrony.

\section{Limitations}

Prevalence studies only, no information on treatment effect.

\section{References}

1. Auricchio A, Abraham WT (2004) Cardiac resynchronization therapy: current state of the art: cost versus benefit. Circulation 109: 300-307.

2. Jarcho JA (2005) Resynchronizing ventricular contraction in heart failure. $\mathrm{N}$ Engl J Med 352: 1594-1597.

3. Tracy CM, Epstein AE, Darbar D, DiMarco JP, Dunbar SB, et al. (2012) 2012 ACCF/AHA.HRS Focused Update of the 2008 Guidelines for Device-Based Therapy of Cardiac Rhythm Abnormalities; A report of the American College of Cardiology Foundation/American Heart Association Task Force on Practice Guidelines. J Am Coll Cardiol 126: 1784-800.

4. Kapetanakis S, Kearney MT, Siva A, Gall N, Cooklin M, et al. (2005) Real-time three-dimensional echocardiography: a novel technique to quantify global left ventricular mechanical dyssynchrony. Circulation 112: 992-1000.

5. Emkanjoo Z, Esmaeilzadeh M, Mohammad Hadi N, Alizadeh A, Tayyebi M, et al. (2007) Frequency of inter- and intraventricular dyssynchrony in patients with heart failure according to QRS width. Europace 9: 1171-1176.

6. Leclercq C, Kass DA (2002) Retiming the failing heart: principles and current clinical status of cardiac resynchronization. J Am Coll Cardiol 39: 194-201.

7. McAlister FA, Ezekowitz J, Hooton N, Vandermeer B, Spooner C, et al. (2007) Cardiac resynchronization therapy for patients with left ventricular systolic dysfunction: a systematic review. JAMA 297: 2502

8. Zareba W, Klein H, Cygankiewicz I, Hall WJ, McNitt S, et al. (2011) Effectiveness of cardiac resynchronization therapy by QRS morphology in the Multicenter Automatic Defibrillator Implantation Trial-Cardiac Resynchronization Therapy (MADIT-CRT). Circulation 123: 1061.

9. Perry R, De Pasquale CG, Chew DP, Aylward PE, Joseph MX (2006) QRS duration alone misses cardiac dyssynchrony in a substantial proportion of patients with chronic heart failure. J Am Soc Echocardiogr 19: 1257-1263. 
Citation: Jain SK, Jain A, Larsen TR, Burke P, Feldman D (2014) Assessing the Prevalence of Mechanical Dyssynchrony with Activity in Patients with Low Ejection Fraction and Narrow QRS at Rest. J Cardiovasc Dis Diagn 2: 138. doi:10.4172/2329-9517.1000138

10. Yu CM, Chan YS, Zhang Q, Yip GW, Chan CK, et al. (2006) Benefits of cardiac resynchronization therapy for heart failure patients with narrow QRS complexes and coexisting systolic asynchrony by echocardiography. J Am Coll Cardiol 48 2251-2257.

11. Bleeker GB, Holman ER, Steendijk P, Boersma E, van der Wall EE, et al. (2006) Cardiac resynchronization therapy in patients with a narrow QRS complex. J Am Coll Cardiol 48: 2243-2250.

12. Beshai JF, Grimm RA, Nagueh SF, Baker JH 2nd, Beau SL, et al. (2007) Cardiac-resynchronization therapy in heart failure with narrow QRS complexes. N Engl J Med 357: 2461-2471.
13. Muto C, Solimene F, Gallo P, Nastasi M, La Rosa C et al. (2013) A randomized study of cardiac resynchronization therapy defibrillator versus dual-chamber implantable cardioverter-defibrillator in ischemic cardiomyopathy with narrow QRS: The NARROW-CRT study. Circ Arrhythm Electrophysiol 6: 538.

14. Ruschitzka F, Abraham WT, Singh JP, Bax JJ, Borer JS, et al. (2013) Cardiacresynchronization therapy in heart failure with a narrow QRS complex. N Eng J Med 369: 1395-1405

15. Zeppenfeld K, Schalij MJ, Bleeker GB, Holman ER, Bax JJ (2006) Left ventricular dyssynchrony results in acute heart failure: are there more patients who benefit from cardiac resynchronization therapy? J Cardiovasc Electrophysiol 17: 101. 